\title{
Visually Impaired Assistive System
}

\author{
Kanchan M. Varpe \\ Sinhgad College of Engineering \\ Department of Computer Engineering \\ Pune University, Pune, India
}

\author{
M.P. Wankhade \\ Sinhgad College of Engineering \\ Department of Computer Engineering \\ Pune University, Pune, India
}

\begin{abstract}
This paper presents visually impaired assistive system (VIAS) which focuses on independent mobility of visually impaired or blind people who suffer in an unknown environment without any manual assistance. This system employs Radio Frequency Identification (RFID) to achieve an objective of identifying certain paths for the user navigation as well as provide certain features such as object recognition, log records of all users' tag access, emergency button and user track information. This proposed system on the user side include a mobile RFID reader module with an integrated microcontroller, zigbee transceiver for transmitting the tag's information and TTS for playing information to the user and on the server side zigbee transceiver for wireless communication. In path identification technique, RFID passive tag network is employed on the path and for object recognition required tools and other objects in the house or building will be embedded with passive RFID tags. A text data unique to each object and path location, resides on the server. The reader reads the tags and transmits the data wirelessly to the server PC which in turn scans for the received Tag ID in the database and respond to the user with its related text data which is played at the user side by converting it from text to speech with the help of TTS module. The feasibility and reliability of the developed system is tested by deploying the proposed system at Blind school of girls at Pune, India. As an overall this system will help visually impaired person to gain the feelings of visualization.
\end{abstract}

\section{General Terms}

Blind navigation system, visually impaired user navigation and object recognition system.

\section{Keywords}

Visually Impaired, Navigation, Recognition, RFID, Zigbee, TTS.

\section{INTRODUCTION}

Moving through an unknown environment becomes a real challenge for the visually impaired or blind people, although they rely on their other senses. An age old mechanism used for assistance of the blind or visually impaired people is a white cane commonly known as walking cane a simple and purely mechanical device to detect the ground, irregular surfaces, holes and steps using simple tactile-force feedback.

Apart from walking cane there are not many systems reported so far to help them by using technology. After a thorough literature survey it is revealed that visually impaired people have always been out off the big companies' scope and successful technological means are yet to be developed which will empower them. The proposed system aims to be a techno-friend of visually impaired people to assist them in the orientation and navigation in their residences or at the necessary environments and also it will provide the assisting features such as object recognition, log records of all users tag access, emergency button and user track information for help.

Some navigation system has proposed a solutions such as by using PDA for the information storage and RFID with the use of Bluetooth connectivity for data transfer [11], Similar work with RFID and GSM to retrieve location information for the blinds [7], and one more proposed solution uses RFID, ZigBee and FM transmitter and receiver for the data transfer [1].This paper presents the VIAS system which is designed to run for a visually impaired or blind people and to allow them to find the way from the current location to the required location within a system implemented environment and to get the details of the objects placed in a path or in a range. The data will be stored at the server database. The system is connected to the VIAS database using a wireless technology such as ZigBee Transceivers for full duplex communication where previously used FM transmitters were restricting the number of users and at the user end Text to Speech module is used to play the related text to the user.

For the rest of this paper, some related work is discussed in Section 2, and programmers design is discussed in Section 3 with respective details. The results and discussion are explained in Section 4. And finally, Section 5 concludes and suggest for future work.

\section{RELATED WORK}

The main encouraging factor for the application of technology for visually impaired people is the policy measures adopted by the western countries for social inclusiveness. Lot of development work in these countries is attributed to the above mentioned policy measures and grants invested for supporting this work. In the year 2003, international conference dedicated to the theme of application of technology for all aspects of sight loss showcased many such devices [6].

There were some notable devices exhibited such as PAN OPTICUS which is placed between a digital satellite receiver and a TV to read the menus on the screen, MONOMOUSE to be fitted with a diffuser in order to reduce glare for reading things like CD or DVD covers for low vision people, SONUS 1XT this is a device for voice readout of the scrolling text of track information provided by the stations, and MOBI-CLICK a device to keep track of a person's movements [14]. Solemn work and surveys have also been undertaken to provide access of electronic resources to the visually impaired people by changing the format of the web sites and URLs [17].

Yet another successful project undertaken was RadioVirgilio/Sesamonet to design and implement a reliable system to assist visually impaired citizen's independent mobility in urban settings. The goal was achieved by integrating traditional assistive technologies with wireless 
PDA and RFID technologies to realize an easy to use navigation system. However the system was not successful, since it failed to integrate with the state of art internet technology [11].

One more successful project undertaken was Blind Path Identification System, this design was based on RFID [7]. This strategy comprises a mechanism that aim at ensuring convenience for the blind, it broadcasts the detected location via voice in order to find the detailed street and store number, which cannot be done by blind tiles. This was achieved by integrating GSM and RFID technologies to realize an intelligent navigation system. However the system was not very successful, since it failed to establish wireless communication.

Some new commercial devices appear on the market, like the Ultra Cane which uses a build-in sonar system and sends back vibrations through the handle according to the presence of obstacles [16]. The ultra cane was an enhancement of the traditional white cane by giving information about the obstacles before direct contact with it. But it doesn't provide any new functionality like localization which is still done by movement of the cane. The majority of the system developed so far focus on maintaining spatial orientation which is a major challenge for people with visual impairment. There is the need of systems for providing blind people with information on where they are, hazards that might be in the way, and a description of what lies in their surroundings [12].The idea of spatial orientation refers to the skill to establish awareness of space position relative to land marks in the surrounding environment [4]. The goal of functional independence to visually impaired people can be achieved by providing references and sorts of land marks to improve awareness of the surroundings [5]. The systems based on similar lines have been reported [2,3] and also patents are also filed in this area of development [8-10].

Recently there is one more successful solution for the visually impaired people as RFAIDE which uses RFID, zigbee and FM transmitter and receiver for the data transfer [1], but it can be analyzed that there are some restrictions which limit the functionality of this solution such as use of FM Transmitter will limit the access because for each blind person there will be requirement of a single FM Transmitter on the server side with allocation of specific Frequency band for it which will limit the number of user. Also given system uses zigbee Transceivers which can be used for both Transmission and as well as for receiving the data, which remains unutilized in this system. This system can cost high due to the increase in requirement of hardware for per multiple user access like FM Transmitters and receivers. The main limitation can be said to be of limited user access to the system.

To overcome these restrictions VIAS solution is proposed to make it more beneficial and functional that is visually impaired assistive system (VIAS) which is similar to that proposed in [1] but here is the use of wireless technology such as zigbee Transceivers for full duplex communication where previously used FM transmitters were restricting the number of users and at the user end Text to Speech module is used to play the related text to the user. In this system all the required information will be stored on the DB server.

\section{BASIC NAVIGATION AND OBJECT RECOGNITION SYSTEM}

The proposed assistive system is composed of three subordinate systems, the pathway infrastructure, the navigation device, and database server as shown in Fig 1. The pathway infrastructure is designed with the grid of RFID tags. Each tag can be fixed under the carpet or floor of the path. The RFID tag is used by visually impaired people for navigation. Alternatively, each tag can be installed at sign position along a pathway. The type of RFID tags is selected upon the usage. Here, the LF passive RFID tags are selected since the radio signal penetrates the floor or carpet surface better. The tag grid can be mounted along the walkway or at least at the junction of the walkway. The tag stores the tag ID, and its respective tag location data at the server.

Similar to the pathway infrastructure, the Object recognition infrastructure is designed. The necessary Object's present in the rout, at the campus, building, corridors, and room or at any other essential area will be identified and then RFID tag has to be placed over an each object. In this case, RFID tag is also used by visually impaired people for object recognition. Same type of RFID tag is selected like pathway. The tag stores the tag ID, and its respective object location with its details data at the server.

This assistive device is an embedded system that is equipped with an RFID reader, a microprocessor unit (MCU), a communication module with Zigbee Transceiver and Text To Speech Module with headphones for providing details to user. The proposed device block diagram is shown in Fig 2. The MCU is DS89C420 with 256 bytes of RAM, 16 Kbytes of Flash memory. The RFID reader is low-cost method for reading passive RFID EM4100 family transponder tags which operate at $125 \mathrm{kHz}$. The RFID Tag's selected are passive tags with low frequency and EM4001 ISO based RFID IC. The reader is connected to MCU via RS-232 Serial port. The reader retrieves the Tag ID data from a tag and transfers it to the MCU for further processing. The communication device used is zigbee. The zigbee communicates with the database server to send a request by sending Tag ID and to receive information from the server for the respective path location and object in access. The VIAS use the RC8660FP TTS module to convey received text information in the form of speech to the user via headphone. The connection to the server is only when the navigation and recognition of objects starts.

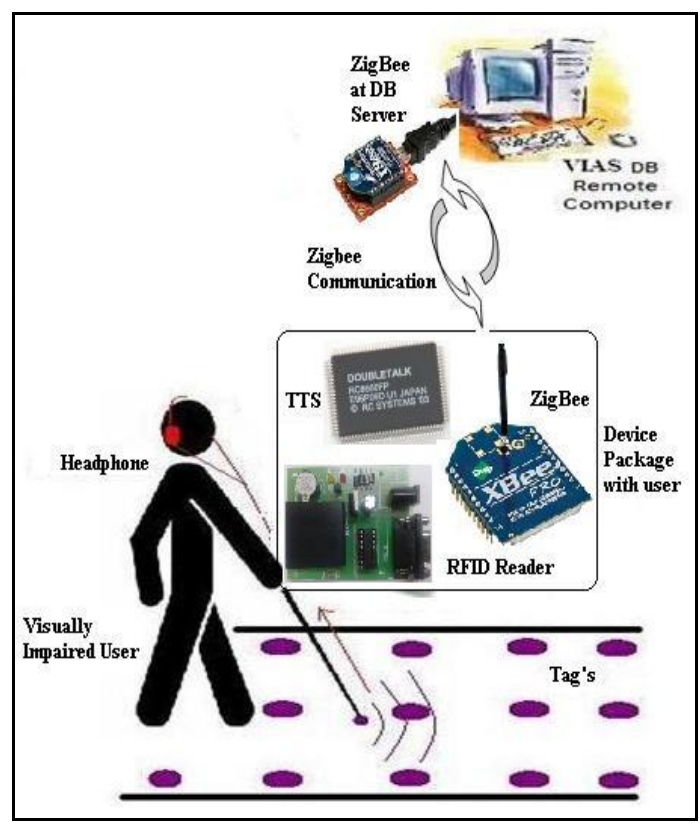

Fig 1: The proposed VIAS system 


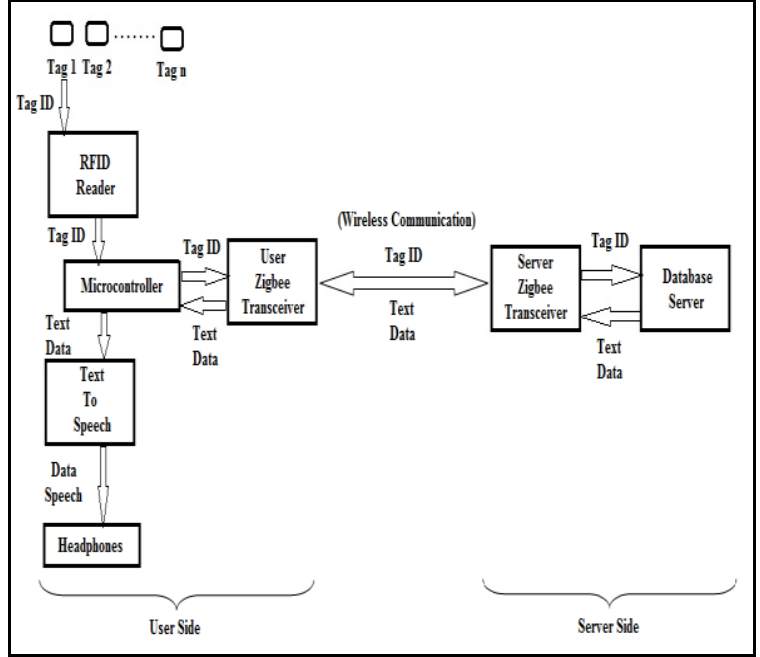

Fig 2: The proposed VIAS device block diagram

\subsection{VIAS working process}

The VIAS consists of a portable and low power cane mounted RFID reader for fetching tag's information and sending it wirelessly to the database server. There are two zigbee transceiver modules for communication which are interfaced with the reader circuit and the database server PC for data transmission. The cane mounted reader reads the Tag ID information stored on the tag and transmits it by using user zigbee to the database server zigbee present in close proximity. On the server side, the received tag ID or unique number is searched in the database and its respective text data is retrieved and this text data is transmitted to the user via server zigbee. The visually impaired user is provided with a Text to Speech module connected to the zigbee transceiver where user zigbee receives text data and provide it to the TTS then TTS covert it from text to speech. In this way user gets the details of pathway location and objects in access. Fig 2 represents the working flow of the VIAS system.

\section{VIAS ASSISTIVE FEATURES}

Independent navigation and object recognition are necessary objective to achieve but with this there is a need to provide certain features so that it will be assistant package for the visually impaired people. As necessary provisions can be provided like maintaining log records of all the users tag access, emergency button for the user when he is in need of manual help and maintaining user track information. These VIAS features are explained in the following sub sections of this section IV and also its experimented results are shown in next section $\mathrm{V}$.

\subsection{VIAS log records}

All the records related to the tag access will be maintained with the help of this feature. The log record will maintain information like User ID, Tag ID accessed, Location of tag, Time of tag Access, etc. This log will be maintained at that time when Tag ID will be received by the DB Server. This feature will help admin to track present and past Tag access by the users. Also admin can perform search operation based on attributes selection.

\subsection{VIAS emergency button}

Overall system will help user to navigate and recognize objects but if user in need of manual help and he wants to call any person for help then this feature will play vital role.
There is one provision of one button over the device present with user. Whenever user wants, he can press the button. After pressing the button microcontroller will send a signal with user ID to the administrator present on the server. Administrator will get a notification that particular user is in need of manual help, then administrator will send any person to help user by finding users location with the help of track map information feature or by checking log records to retrieve recent tag location accessed by the user.

\subsection{VIAS user track information}

The user track information will show track followed by each user. So that administrator will be known to the path followed by the user to send them manual help or to give alert according to the situations caused at the respective locations.

\section{EXPERIMENTS AND RESULTS}

In this section, actual system prototype is explained and results discussion of Visually Impaired Assistive System is done on the basis of experimental execution conducted with the help of two walkthrough sessions.

\subsection{VIAS prototype}

The VIAS system prototype includes a simulated map of 30 tags to form a grid over the path. Out of 30 Tags, 20 tags contain Tag ID and its path location details and remaining 10 tags contain Tag ID and attached objects details. All the tags are placed at the necessary path location and at the objects. After placing the tags VIAS database is updated with Tag ID and details associated with each Tag. Here, software facility is provided for the DB maintenance with authorized access to it where general user can perform search and view the data but cannot perform any other operation whereas an administrator has to do all the operation on the DB such as insert, delete, update, save, search etc.

The prototype of the VIAS device is shown in Fig. 5. The actual size of the prototype is about $12 \times 18 \times 6 \mathrm{~cm}$ in dimension and about $0.7 \mathrm{~kg}$ by weight, not including the tag reader. The tag reader is mounted on stick. The device is portable and equipped with a headphone and speaker where voice will guide the users for navigation and recognition. The device is operated by a rechargeable $12 \mathrm{~V}$ battery that can last about 6 hours. The device is attached to a user as shown in Fig 3 with a navigation cane for blind or visually impaired people.

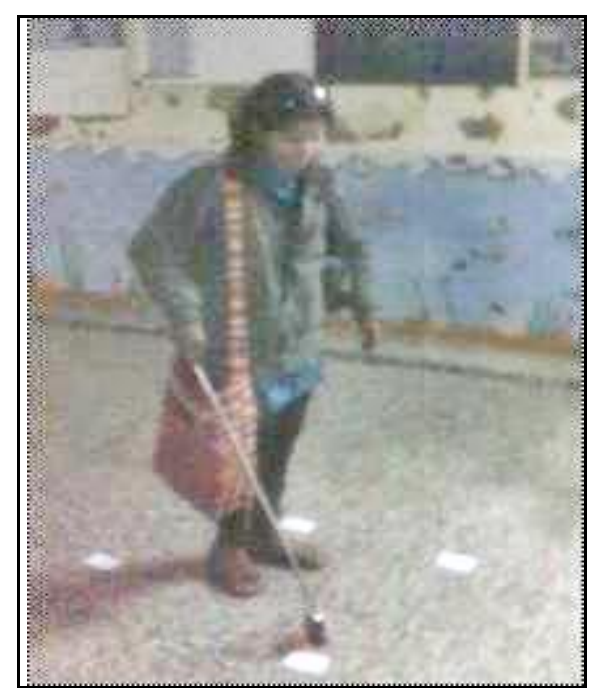

Fig 3: The device using blind user 


\subsection{Experiments and results discussion}

This system is tested by a simulation of the navigation and recognition. Testing of this device is done at the two locations first at the laboratory of the engineering department and secondly at the Poona School \& Home for the Blind, Pune, India.

The testing is performed through two walkthrough sessions in order to talk about the system's basic functionalities and to emphasize the most critical characteristics to be updated or to be tested with users. First walkthrough session were participated by author plus some faculty members and friends at Computer Laboratory of College, while second walkthrough session was participated by the blind users at the Blinds School of Girls, Pune.

In all the walkthrough sessions one or more participants executed some basic tasks within a typical scenario while the other members observed this execution. Each member was taught about the system responses and the tags character in the path or on the object; they were then trained to move toward the VIAS walkway by scanning with the cane and moving on, keeping the cane at suitable distance from the floor and trying to find a path over it.

While execution of the VIAS, when a user is at walkway and start walking then according to the tags placed on the path, device describes the path location in the voice form such as "Entrance of Computer Laboratory" and for the object in access it gives message such as "Object is Computer Table", likewise messages are provided to the user.

Now, the features implemented with base system as described above are also tested. All the software part is implemented with the help of VB.Net in Microsoft Visual Studio 2008 and DB used is MySQL 2008. All the features are working well such as for the Log Record Maintenance which is triggered as soon as tag is accessed and stores its necessary record with required attributes also supports search operation.

The main tasks of this system are sending and receiving procedure for data which is as shown in Fig 4.

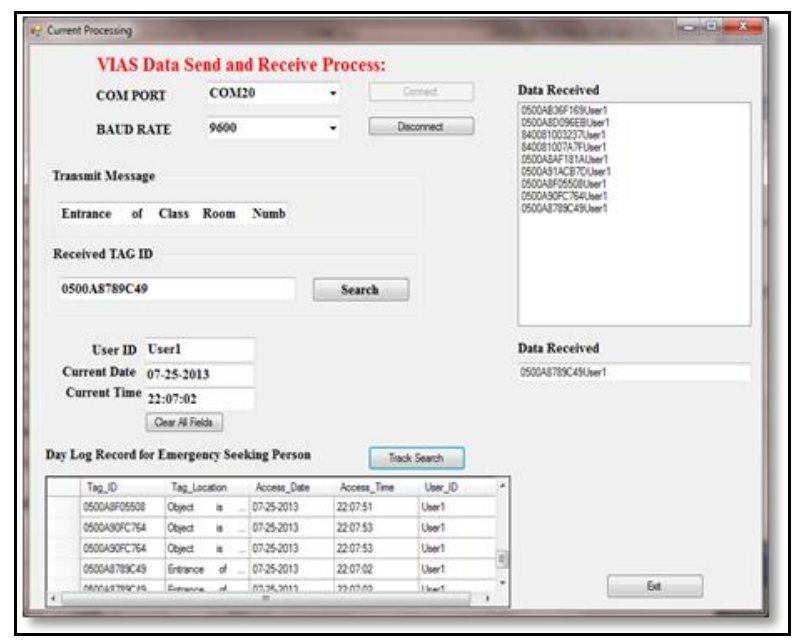

Fig 4: VIAS data send and receive process

The Emergency Button feature provides best result by sending signal with user ID to the server so that admin can send a manual help to visually impaired user. The circuitry device for this feature is shown in Fig 5 and Fig 6 with server response to the emergency button.

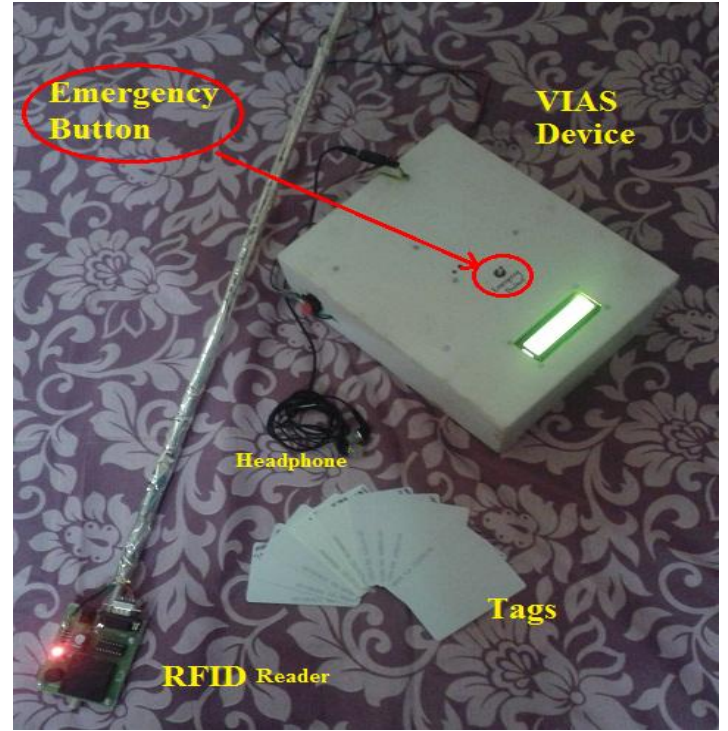

Fig 5: VIAS emergency button on the user device

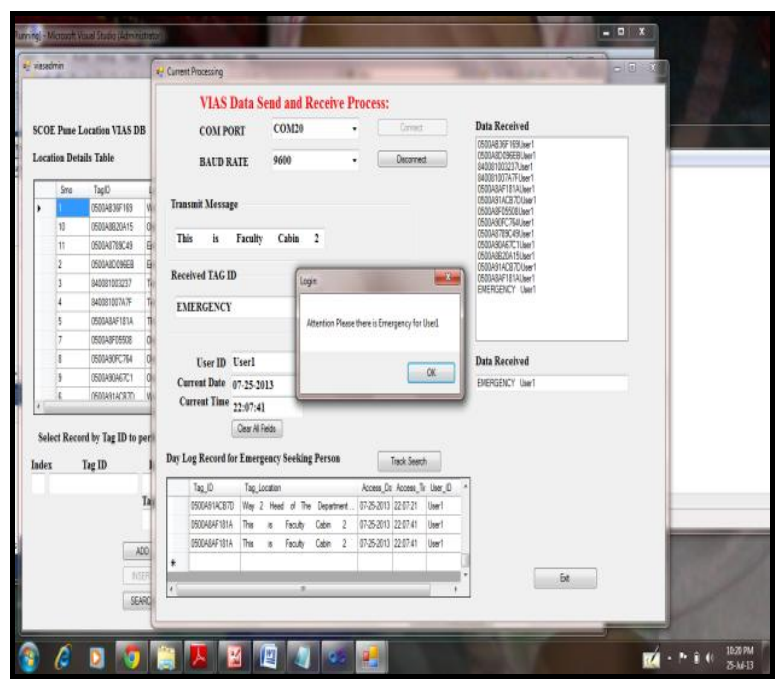

Fig 6: VIAS emergency button response at server

In case of last feature of User Track Information, provides all the details according to the user which help us to know the track of user. By using this track admin can send immediate help to the user by following the same track followed by the user. The results of this feature are shown in Fig 7.

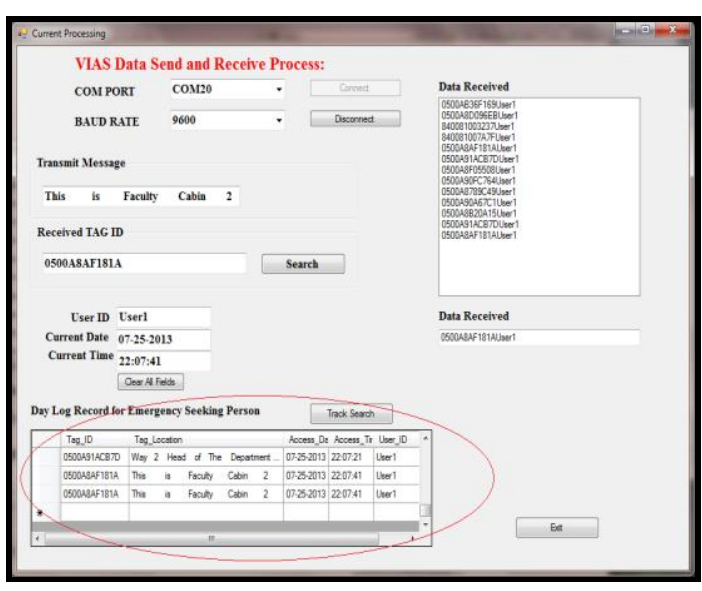

Fig 7: The VIAS user track information 
Also following Table 1 describes the overall Tag access results while an execution of the VIAS.

Table 1. Tag access results

\begin{tabular}{|c|c|c|}
\hline $\begin{array}{l}\text { Sr. } \\
\text { No. }\end{array}$ & Case Details & Execution Results \\
\hline 1. & $\begin{array}{l}\text { Reader faced } \\
\text { exactly to Tag }\end{array}$ & Exact Data retrieved \\
\hline 2. & $\begin{array}{l}\text { Reader moved } \\
\text { over the Tag }\end{array}$ & $\begin{array}{l}\text { Sometimes Tag is } \\
\text { detected and exact data } \\
\text { is retrieved but } \\
\text { sometimes Tag is not } \\
\text { detected }\end{array}$ \\
\hline 3. & $\begin{array}{l}\text { Two Tags faced } \\
\text { to the Reader }\end{array}$ & $\begin{array}{l}\text { Any one Tag is detected } \\
\text { with its exact data } \\
\text { retrieval. }\end{array}$ \\
\hline 4. & $\begin{array}{lr}\text { Location } & \text { or } \\
\text { Object } & \text { Details } \\
\text { retrieval } & \\
\end{array}$ & $\begin{array}{l}\text { the majority results are } \\
\text { correct }\end{array}$ \\
\hline 5. & Tag detection & $\begin{array}{l}\text { the majority correct } \\
\text { data is retrieved but few } \\
\text { times it has given a } \\
\text { error message as } \\
\text { location not found }\end{array}$ \\
\hline 6. & $\begin{array}{l}\text { Emergency } \\
\text { Button }\end{array}$ & $\begin{array}{l}\text { Once pressed it gives } \\
\text { twice notifications to } \\
\text { admin system with } \\
\text { emergency music in } \\
\text { background. And also } \\
\text { after pressing Track } \\
\text { button shows Tags } \\
\text { accessed by the user. }\end{array}$ \\
\hline 7. & $\begin{array}{l}\text { Zigbee to Zigbee } \\
\text { Communication }\end{array}$ & $\begin{array}{l}\text { Delay or barrier is } \\
\text { experienced in the } \\
\text { presence of hard } \\
\text { obstacles or in indoor } \\
\text { environment. But at } \\
\text { outdoor location it } \\
\text { works effectively. }\end{array}$ \\
\hline
\end{tabular}

These are the results achieved after analyzing the experimental execution of visually impaired or blind people assistive system.

\section{CONCLUSION AND FUTURE WORK}

The guide system for visually impaired people is presented in this paper which is an assistive system with the help of RFID, Zigbee and TTS for blind or visually impaired people to help them for navigation and object recognition with assistive features. The VIAS can be used by visually impaired users at the system implemented environment such as organization campus which can be school, college, hospitals, shopping mart, bus stands, markets etc. A VIAS system prototype includes RFID tag grid embedded under the walkway and placed over the objects, the embedded system as a navigation and recognition device, and a database server which is remotely connected to the user device via zigbee communication. The VIAS system prototype has shown the promising result although its size is still large and giving service to multiple persons. This system uses low data rate as it transfers text data instead of audio file.
Also, there is some communication delay between the device and server. This is due to the hard obstacles present in the way of communication or caused due to the large distance between user and server zigbee. This problem can be resolved by using network of number of zigbee devices.

In future, task will be focused to decrease the problems related to the communication. Additionally, new feature personal assistance will introduced, which will ask user for the destination location and then it will direct the user according to the shortest path available from the current location to the destination also this feature will help to correct the lost path of the user to direct him exactly to the destination location.

\section{ACKNOWLEDGMENT}

The authors wish thank to all the referees involved in the above mentioned work of VIAS survey. This project is supported by the AICTE for RPS project (File Ref No. 8023/RID/RPS/-132(Pvt)/2011-12). Finally, an honorable mention goes to our family and Prof. Mahendra Deore and Mr. Manohar Varpe for their understandings and supports on this project.

\section{REFERENCES}

[1]. Abdullah Rehman, Mohsin Murad, Arif Ali Shah, Salim Ullah, Muhammad Fahad, Khawaja M. Yahya , "RFAIDE--An RFID Based Navigation and Object Recognition Assistant for Visually Impaired People", University of Engineering and Technology, Peshawar, Pakistan, 978-1-4577-0768-1/11/2011 IEEE.

[2]. Andreas Hub, Joachim Diepstraten, Thomas Ertl, "Design and Development of an Indoor Navigation and Object Identification System for the Blind", Visualization and Interactive Systems Institute University of Stuttgart.

[3]. Abdelsalam (Sumi) Helal, Steven Edwin Moore, Balaji Ramachandran, "Drishti: An Integrated Navigation System for Visually Impaired and Disabled", University of Florida,Gainesville, FL-32611.

[4]. Guth, D.A.; Rieser, J.J. "Perception and the control of locomotion by blind and visually impaired pedestrians". Foundations of Orientation and Mobility, (Second Edition), AFB Press, pp. 9-38, 1997.

[5]. H. Mori, S. Totani, "Robotic Travel Aid for the Blind: HARUNOBU-6". In Proceedings of the Second European Conference On Disability, Virtual Reality, and Assistive Technology, Sövde, Sweden, 1998.

[6]. Jenny Crave, Research Fellow, "Access to electronic resources by visually impaired people", CERLIM, Manchester Metropolitan University, UK, Information Research, Vol. 8 No. 4, July 2003.

[7]. Jinying Chen, Xuben Wang, Zhi Li, Min Dong, "Blind Path Identification System Design Base on RFID", 2010 International Conference on Electrical and Control Engineering.

[8]. L. W. Alonzi, D. C. Smith, G. J. Burlak, M. Mirowski, (1992). "Radio frequency message apparatus for aiding ambulatory travel of visually impaired persons", U.S. Patent 5,144,294 issued Sept. 1, 1992.

[9]. M.B. Hancock, "Electronic auto routing navigation system for visually impaired persons". U.S. Patent 5,806,017 issued September 8, 1998. 
[10].T. E. Piotrowski, "RFID navigation system". EP Patent 1 $313079,2003$.

[11].Ugo Biader Ceipidor, Graziano Azzalin, Mariangela Contenti,"A RFID System to Help Visually Impaired People in Mobility", published in proceedings of Second International Workshop on Mobile Communications and Learning, Martinica, 22-29 April 2007.

[12].W.C. Mann, "The aging population and its needs", IEEE Pervasive Computing, Vol.3, No. 2, April-June 2004, pp. 12- 14.

[13].Willis, S. Helal, S. Comput., "RFID information grid for blind navigation and wayfinding," in Proceedings of Ninth IEEE International Symposium on Wearable Computers, 2005.

[14].Survey information [Online] Retrieved on July 10, 2012 :http://news.bbc.co.uk/2/hi/technology/4412283.stm.

[15].Text to Speech [Online] accessed on 15 January 2013, http://www.rcsys.com/dnlds.htm\#docs.

[16].Ultracane Itexttrademark, www.soundforesight.co.uk. Retrieved on August 3,2012.

[17].Visually impaired see the future by Geoff Adams-Spink, BBC News website disability affairs correspondent, http://news.bbc.co.uk/1/hi/technology/4412283.stm Retrieved on August 25, 2007.
[18].XBee Modules, Digi International, [Online] accessed on August 16, 2012:http://www.digi.com/products/wirelesswired-embedded-solutions/zigbee-rf-modules/zigbeemesh-module/xbee-digimesh-2-4.jsp.

\section{Survey Paper:}

[19].Kanchan Varpe, M.P. Wankhade, "Survey of Visually Impaired Assistive System", published in International Journal of Engineering and Innovative Technology (IJEIT) Volume 2, Issue 11, May 2013

\section{ABOUT THE AUTHORS}

Kanchan M. Varpe received the B.E. degree in Computer Engineering from Pune University, Maharashtra, India in 2007 and the M. E. degree in Computer Network (Computer Engineering) at Sinhgad College of Engineering from Pune University, Maharashtra, India in 2013. Her area of interest is RFID, DBMS, and Image Processing. She has teaching experience of 6 years and currently working as Asst. Professor.

M. P. Wankhade received the M.Tech. degree in Information Technology from Bharati Vidyapeeth, Maharashtra, India and He has teaching experience of 17 yrs and currently working as Associate Professor at Sinhgad College of Engineering, University of Pune, Maharashtra, India. His area of interest is Image Processing. 